\title{
A new endocapsular open ring for prevention of anterior and posterior capsule opacification
}

\author{
This article was published in the following Dove Press journal: \\ Clinical Ophthalmology \\ 3 November 2016 \\ Number of times this article has been viewed
}

\author{
loannis G Pallikaris \\ Nela R Stojanovic \\ Harilaos S Ginis \\ Institute of Vision and Optics, \\ Department of Medicine, University \\ of Crete, Heraklion, Crete, Greece
}

Purpose: The aim of this study is to demonstrate the functionality of a new design of a thick endocapsular open ring for prevention of anterior capsule opacification (ACO) and posterior capsule opacification (PCO).

Setting: The Institute of Vision and Optics, University of Crete and University Hospital of Heraklion, Crete, Greece.

Design: Prospective, interventional pilot study.

Methods: Fifteen patients (17 eyes) underwent cataract surgery with phacoemulsification. During surgery, a thick endocapsular open ring (peripheral capsule reconstructor) was inserted into the capsular bag, prior to intraocular lens (IOL) implantation. Six different models of IOL were implanted. Postoperatively, the degree of ACO and PCO was evaluated and described as none, mild, moderate, or severe.

Results: The mean follow-up period was 30 \pm 8.06 months (range: $12-36$ months). At the last follow-up, mild PCO was observed in only three eyes and mild ACO in three patients. The centration of IOLs was good in all but one eye, which had a tilted IOL.

Conclusion: The results of this pilot study suggest that the implantation of a new design of thick endocapsular open ring is feasible and may contribute to the prevention of PCO and ACO after cataract surgery.

Keywords: intraocular implant, cataract, phacoemulsification, silicone

\section{Introduction}

Modern cataract surgery with phacoemulsification is considered one of the safest surgical procedures in medicine. However, there may be some complications. The most frequent, long-term complication of cataract surgery is posterior capsule opacification (PCO), also known as secondary cataract. ${ }^{1}$ PCO may cause light scattering, monocular diplopia, decrease of contrast sensitivity, and visual acuity. ${ }^{2,3}$ Depending on severity, PCO may significantly interfere with optic results of cataract surgery, especially with premium intraocular lenses (IOLs), such as multifocal and accommodating IOLs.

The common therapeutic modality for PCO is Nd:YAG laser capsulotomy, which consists of clearing the visual axis by creating a central opening in the posterior capsule. Even though this procedure is easy and effective in majority of the cases, there are some disadvantages to it. The Nd:YAG capsulotomy is associated with a number of complications, such as retinal detachment, IOL damage and subluxation, corneal edema, elevation of intraocular pressure, and cystoid macular edema. ${ }^{4-7}$ While some of these complications are mild and transient, other may cause permanent visual impairment.

Another type of capsular opacity is anterior capsule opacification (ACO), also termed anterior capsule fibrosis or anterior subcapsular opacification. ${ }^{1}$ It occurs much earlier and more often compared with PCO, sometimes within 1 month postoperatively.
Correspondence: loannis G Pallikaris Institute of Vision and Optics, Department of Medicine, University of Crete, 71003 Heraklion, Crete, Greece Email pallikar@med.uoc.gr 
Severe forms of ACO may induce contraction of the capsulorhexis opening, capsule retraction, and capsular phimosis. This may cause decentration of the IOL and fibrotic opacification of the posterior capsule. Furthermore, it may compromise visualization of the peripheral retina, especially during retinal examinations and vitreoretinal surgery.

Improvements in cataract surgery technique, IOL materials, and designs have reduced the incidence of PCO and ACO, but the problem still exists. Researchers all over the world are still attempting to eliminate the problem of PCO development, focusing on several strategies, including surgical technique, IOL material, IOL design, biological and pharmacological agents, and intraocular endocapsular rings. ${ }^{8,9}$

The idea of ring insertion into the capsular fornix was introduced by Hara et al in the context of zonular apparatus support. ${ }^{10}$ Two years later, the first capsular tension ring (CTR) was designed for use in humans. Since then, various similar CTRs have been designed and marketed. ${ }^{11}$ The basic characteristic of these endocapsular rings is the ability to maintain the capsule's contour and stretch the posterior capsule. Although CTRs have well-established use for the management of zonular weakness and dehiscence, enhancement of IOL centration, and capsule stabilization, they may also influence capsule opacification. ${ }^{12}$

We report our initial experience with a thick endocapsular open ring with a new design to prevent PCO and ACO.

\section{Patients and methods}

This prospective, interventional pilot study enrolled 17 eyes of 15 patients with senile cataract who were examined and scheduled for routine cataract surgery between January and September 2011 at the Institute of Vision and Optics, Crete, Greece.

Approval was obtained from the institutional review board of the Institute of Vision and Optics, University of Crete, and all patients were appropriately informed before their participation in the study about the possible outcomes and the current clinical experience, and provided written informed consent in accordance with the institutional guidelines, according to the Declaration of Helsinki.

Inclusion criteria were senile cataract and good pupil dilatation. Exclusion criteria were traumatic cataract, severe disease or predisposition of severe disease of anterior or posterior segment, and intraoperative complications that might compromise the integrity of the capsular bag.

The preoperative data obtained from the case records included patient age, sex, slit-lamp biomicroscopy, intraocular pressure, fundoscopy, and endothelial cell count.
The visual parameters measured were uncorrected distance visual acuity and corrected distance visual acuity (CDVA), using the Early Diabetic Retinopathy Study logMAR charts (Precision Vision, IL, USA). The IOL power was calculated with the IOL Master 500 (Carl Zeiss Meditec AG, Jena, Germany). Six different types of IOL were randomly inserted: three-piece IOL Lenstec Inc. Softec III (Lenstec, St Petersburg, FL, USA); one-piece IOL SN60WF Alcon; toric IOL SN60T9 Alcon (Alcon, Fort Worth, TX, USA); Bausch \& Lomb Akreos MI60 (Bausch \& Lomb, Rochester, NY, USA); Morcher 89F (Morcher, Stuttgart, Germany); and WIOL-CF ${ }^{\circledR}$ (Medicem, Kamenné Žehrovice, Czech Republic). The choice of IOL type was random.

Postoperatively, the patients were examined at 1 day, 1, 3, 6, 9 months, and 1, 2, and 3 years. The PCO and ACO formation were evaluated by the same experienced examiner (NS). The development of PCO and ACO was evaluated subjectively on slit-lamp examination with retroillumination and digital photo documentation after pupil dilation. The degree of ACO and PCO was classified as none, mild, moderate, and severe.

IOL centration was visually assessed by slit-lamp examination. The lens centration was considered good when its edge was fully covered by the undilated pupil and its position was not considered to limit visual performance in general.

\section{New design of an endocapsular open ring - peripheral capsule reconstructor}

The peripheral capsule reconstructor (PCR) ring (Figure 1) is a thick endocapsular open ring, designed to maintain contact with the inner surface of peripheral part of the capsule with minimal tension and provides spacing between the anterior and the posterior surface of the capsule. With these design principles, it is intended to prevent endothelial cell migration, to provide a consistent bed for IOL implantation and to prevent fibrosis.

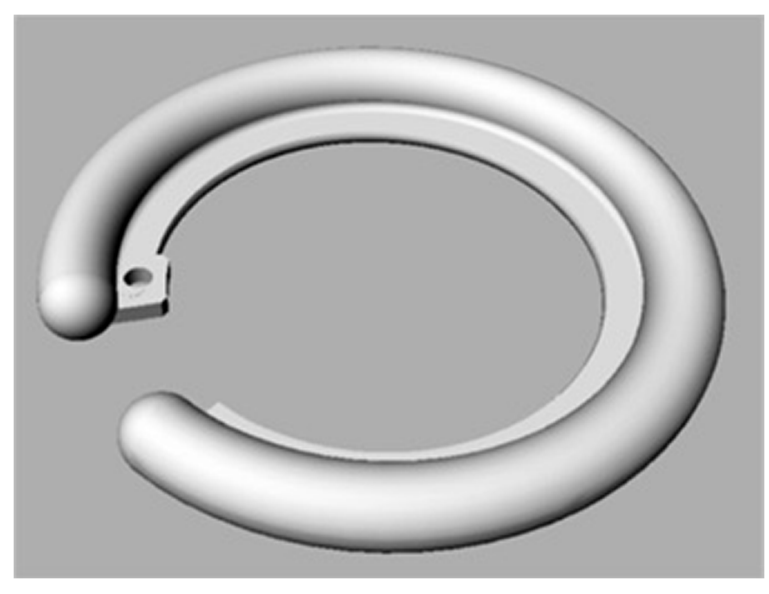

Figure I The design of the thick endocapsular open ring. 
The PCR ring is made of medical grade silicone elastomer (MED-4765, NuSil Technology LLC, Carpinteria, CA, USA). It has an external diameter of $10.5 \mathrm{~mm}$ and a cross sectional diameter of $1.2 \mathrm{~mm}$. It features an internal groove to accommodate different IOL haptics. A lid adjacent to the groove and implanted anteriorly facilitates the insertion of the IOL haptics in the groove. The PCR ring has an opening of $2 \mathrm{~mm}$ that allows reducing its diameter when implanted in smaller capsules.

\section{Surgical technique}

All surgeries were performed by the same surgeon (IGP) at University Hospital of Heraklion, Crete. The surgeries were performed in a standard manner of phacoemulsification under topical anesthesia. After a $2.8 \mathrm{~mm}$ self-sealing clear corneal incision was made, continuous curvilinear capsulorhexis of $5 \mathrm{~mm}$ diameter was created. After phacoemulsification and irrigation/aspiration of the lens cortex, the capsular bag was filled with sodium hyaluronate. The endocapsular ring was then inserted into the capsular bag with one of the commercially available IOL injectors (Alcon Monarch II injector and C cartridge). Finally, the IOL was inserted into the capsular bag and placed in the groove of the ring.

All patients received antibiotic/steroid eye drops (tobramycin/dexamethasone, Tobradex; Alcon Laboratories, Inc., Fort Worth, TX, USA) four times a day for 1 month postoperatively.

\section{Results}

Patient demographics are presented in Table 1.

This study comprised 15 patients (17 eyes). Eight patients were females. The mean follow-up period was $30 \pm 8.06$ months (range: 12-36 months).

Table I Patient demographics

\begin{tabular}{ll}
\hline Demographic & Value (mean \pm SD) \\
\hline Number of patients (eyes) & $15(17)$ \\
Eye with the ring R/L & $7 / 10$ \\
Age (years) & $62 \pm 13.04$ \\
Sex M/F & $7 / 8$ \\
UDVA (logMAR) & $0.68 \pm 0.23$ \\
CDVA (logMAR) & $0.42 \pm 0.16$ \\
IOP (mmHg) & $13 \pm 2.57$ \\
ECD (cells/mm $\left.{ }^{2}\right)$ & $2,011 \pm 189$ \\
Axial length (mm) & $21.67 \pm 1.60$ \\
IOL power (D) & $24.31 \pm 2.90$ \\
Follow-up period (months) & $30 \pm 8.06$ \\
\hline
\end{tabular}

Abbreviations: CDVA, corrected distance visual acuity; ECD, endothelial cell density; IOL, intraocular lens; IOP, intraocular pressure; M/F, male/female; R/L, right/left; SD, standard deviation; UDVA, uncorrected distance visual acuity.
At last follow-up, the mean uncorrected distance visual acuity changed from $0.68 \pm 0.23 \log$ MAR (range: $1.20-0.30$ ) preoperatively to $0.12 \pm 0.08 \log M A R$ (range: $0.00-0.30$ ). The mean CDVA improved from $0.42 \pm 0.16 \log$ MAR (range: $0.20-0.70$ ) preoperatively to $0.02 \pm 0.03 \log$ MAR (range: $0.00-0.10$ ). No patient lost lines of CDVA. Endothelial cell count changed from $2,265 \pm 209$ preoperatively to $2,011 \pm 189$ at last follow-up examination. Intraocular pressure changed from $13 \pm 2.57$ to $14 \pm 2.40 \mathrm{mmHg}$.

Six different models of IOL were implanted: three-piece Lenstec Inc. Softec III IOL was inserted in six eyes, one-piece Alcon SN60WF in four, WIOL-CF ${ }^{\circledR}$ in two, Morcher 89F in three, B\&L MI60L in one, and toric SN60T9 in one eye. No intraoperative complications occurred.

On day 1 postoperatively, mild anterior chamber glare was observed in two eyes (10\%). After administration of a short course of topical steroid therapy $(0.1 \%$ dexamethasone eye drops six times per day with a tapering regime of one drop every week), the inflammation resolved completely, with no residual abnormality. In one eye, the IOL was tilted, with one haptic positioned in the gap of the ring. During the follow-up examinations, no other postoperative complications of any kind were observed.

At last follow-up, only in three eyes was observed mild (not likely to cause visual disturbance) PCO (Table 1). One eye had tilted IOL, with one haptic placed into the ring gap (Figure 2) and developed PCO on 50\% surface of the IOL optic. In the second eye, at 12 months postoperatively, mild PCO developed, located peripherally at the gap, far from

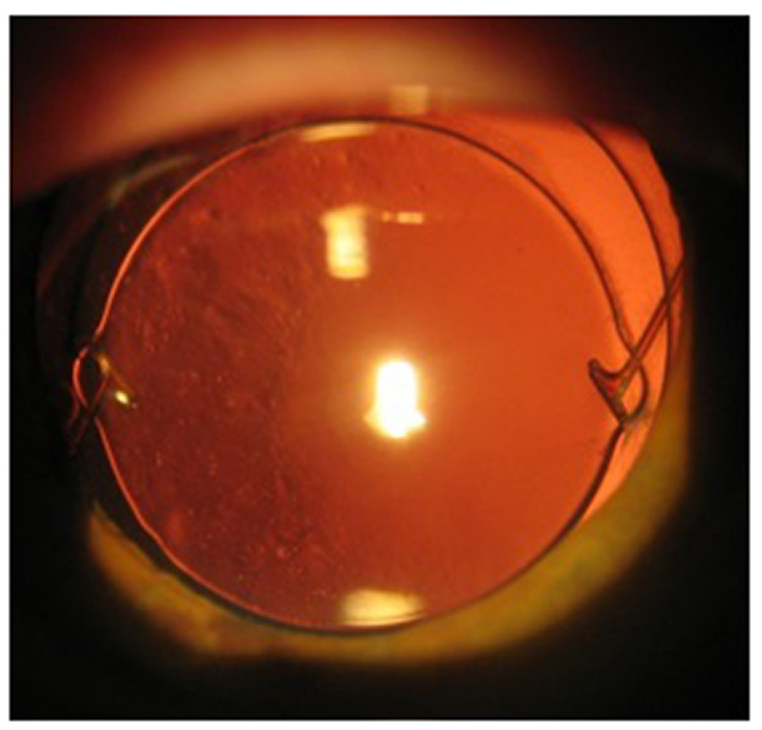

Figure 2 The slit-lamp retroillumination photo of PCO in eye with tilted IOL and one haptic located in the ring gap.

Abbreviations: IOL, intraocular lens; PCO, posterior capsule opacification. 

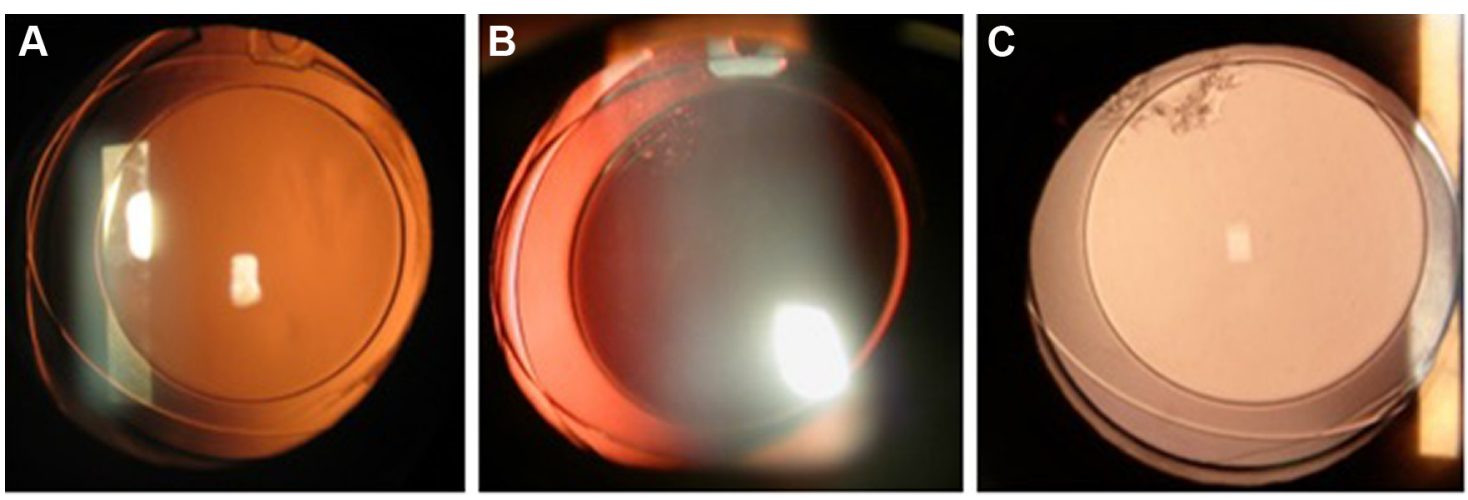

Figure 3 The slit-lamp retroillumination photos of peripheral PCO development at the ring gap in the same eye at 3 (A), 12 (B), and 36 (C) months postoperatively. Abbreviation: PCO, posterior capsule opacification.

the optic center. The PCO surface size remained the same at follow-up intervals, but the morphology had changed between 12 and 36 months postoperatively (Figure 3). The third eye had mild capsule opacification from day 1 postoperatively that did not change in density or size until the last follow-up. In one patient who underwent bilateral cataract surgery with IOL implantation, but had the ring inserted in only one eye, a significant difference in PCO formation between eyes was observed at the 24-month follow-up (Figure 4). Mild ACO was observed in three patients at last follow-up (Figure 5). The centration of IOLs was good in all but one eye with tilted IOL.

In one patient with WIOL implantation in her right eye, an exchange of IOL was performed 12 months after cataract surgery. Before the IOL exchange, the patient had CDVA of 20/32 and was not happy with her vision. The WIOL was removed from the capsular bag, without taking out the ring, and another type of IOL (monofocal three-piece IOL,
Softec, Lenstec Inc.) was placed inside the capsular bag in the grooves of the ring. Four months postoperatively, her CDVA improved to 20/20 and the capsular bag remained clear until the last follow-up at 36 months (Figure 6).

\section{Discussion}

Capsule opacification is essentially caused by lens epithelial cells (LECs) left behind the capsular bag after extracapsular cataract extraction. Possibly surgical trauma induces woundhealing response in the residual LECs, which manifests through proliferation, migration, epithelial-to-mesenchymal transition, collagen deposition, and lens fiber regeneration. ${ }^{1}$ Ultimately, LECs may cover the visual axis, resulting in significant capsular opacification and light scattering. Beside this direct blockage of visual axis, severe proliferation of LECs can also indirectly, through mechanical forces, cause complications, such as posterior capsule striae or folds or traction-induced IOL malposition. Regardless of the
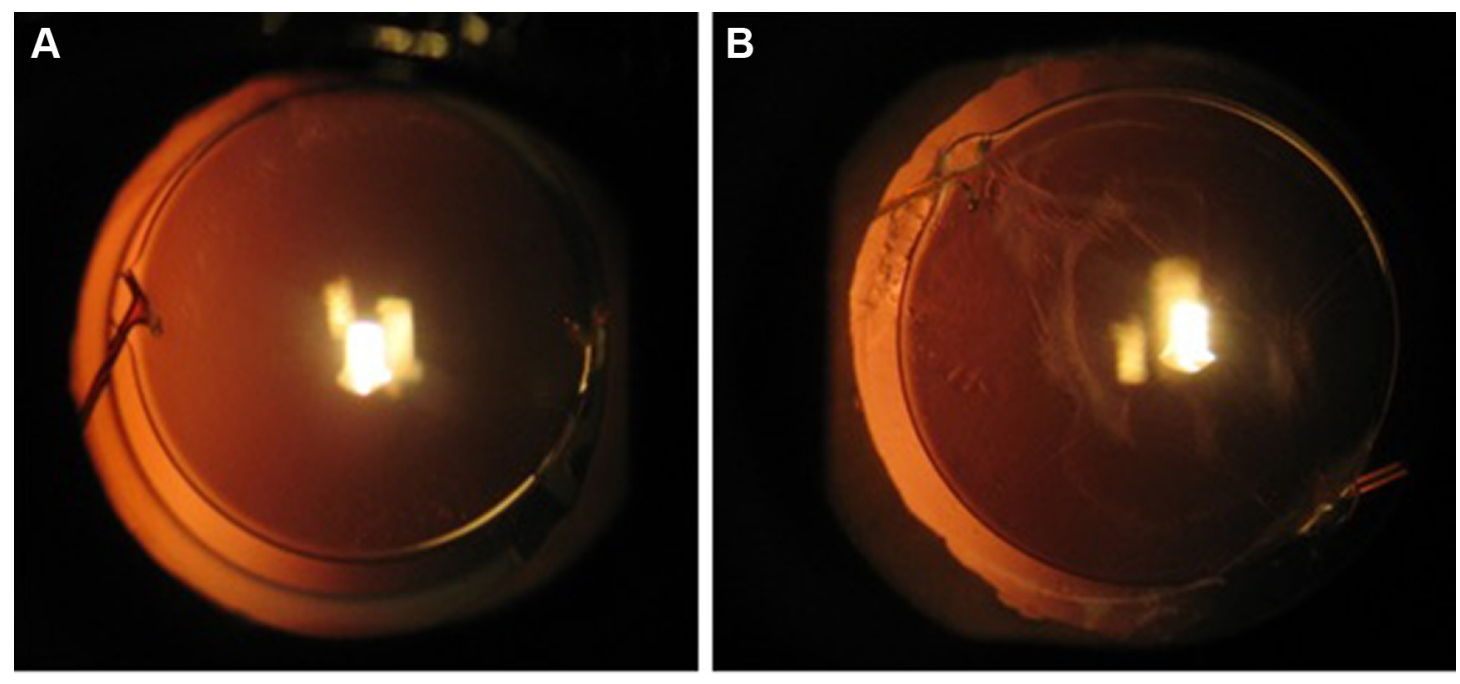

Figure 4 The slit-lamp retroillumination photo of the right eye $(\mathbf{A})$ with the ring and left eye $(\mathbf{B})$ without the ring 36 months postoperatively in the same patient. 


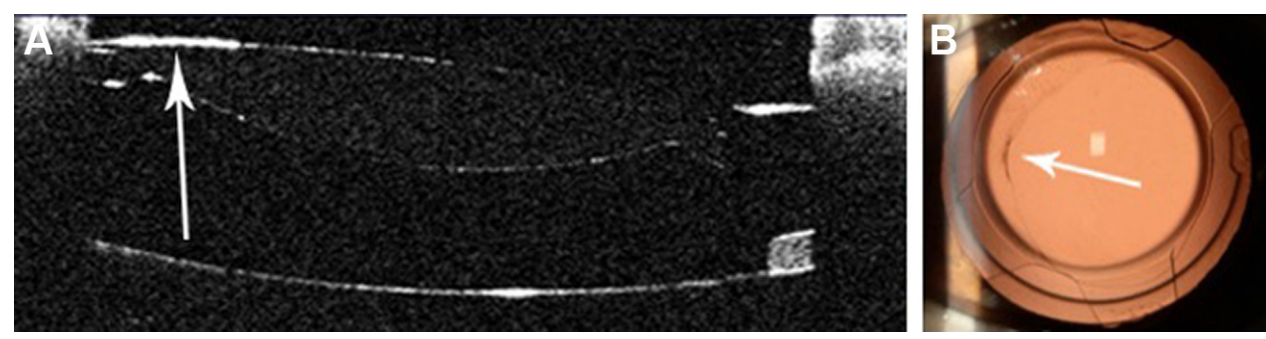

Figure 5 The anterior segment optical coherence tomography $(\mathbf{A})$ and slit-lamp (B) photos of anterior capsule wrinkling of the same patient (arrows).

mechanism, both $\mathrm{PCO}$ and $\mathrm{ACO}$ may result in the decrease of visual acuity, which, if severe, may considerably reduce the quality of the patient's vision.

The first capsular rings were designed as capsule support devices in cases of zonule rupture or weakness. ${ }^{11}$ It was only later observed that these rings have an effect on capsule opacification. Since then, several capsular rings have been studied in the context of PCO prevention.

The closed sharp-edged silicone equator ring (E-ring) was first studied for its efficiency in $\mathrm{PCO}$ prevention in rabbit eyes. ${ }^{13}$ Long-term results in human eyes were very encouraging, showing significantly reduced central PCO in the eyes implanted with the ring in comparison to a control group. ${ }^{14}$ The authors suggested that the E-ring of $9.5 \mathrm{~mm}$ diameter suits all eyes, based on their previous observation of postoperative iritis in eye with a $9.0 \mathrm{~mm}$ ring, probably caused by rotation of the ring in the capsular bag. The E-ring is contraindicated for eyes with disorders of the posterior capsule and Zinn ligaments and requires the use of a specially designed IOL.
A different model of the endocapsular ring is an open ring made of polymethylmethacrylate with a rectangular profile, termed as a capsular bending ring (CBR). The study results of 3-year follow-up showed that objective PCO score and area were statistically significantly reduced in CBR eyes when compared to the no-CBR group. ${ }^{12}$ The barrier failure in some eyes with a CBR was explained by inherent slight edge blunting due to the ring material characteristics and occasional ring eyelet gaping.

Separation of the anterior capsule from the IOL optic's anterior surface and the posterior capsule inhibits epithelialto-mesenchymal transition and fibrous metaplasia of the LECs. Thus, the anteroposterior capsule separation not only prevents whitening and contraction of the anterior capsule leaf, but also fibrotic outgrowth onto the posterior capsule and epithelial growth onto the anterior IOL surface. The importance of the separation and aqueous humor (AH) circulation in prevention of $\mathrm{PCO}$ was discussed in the study with a capsular adhesion-preventing ring (CAPR) introduced
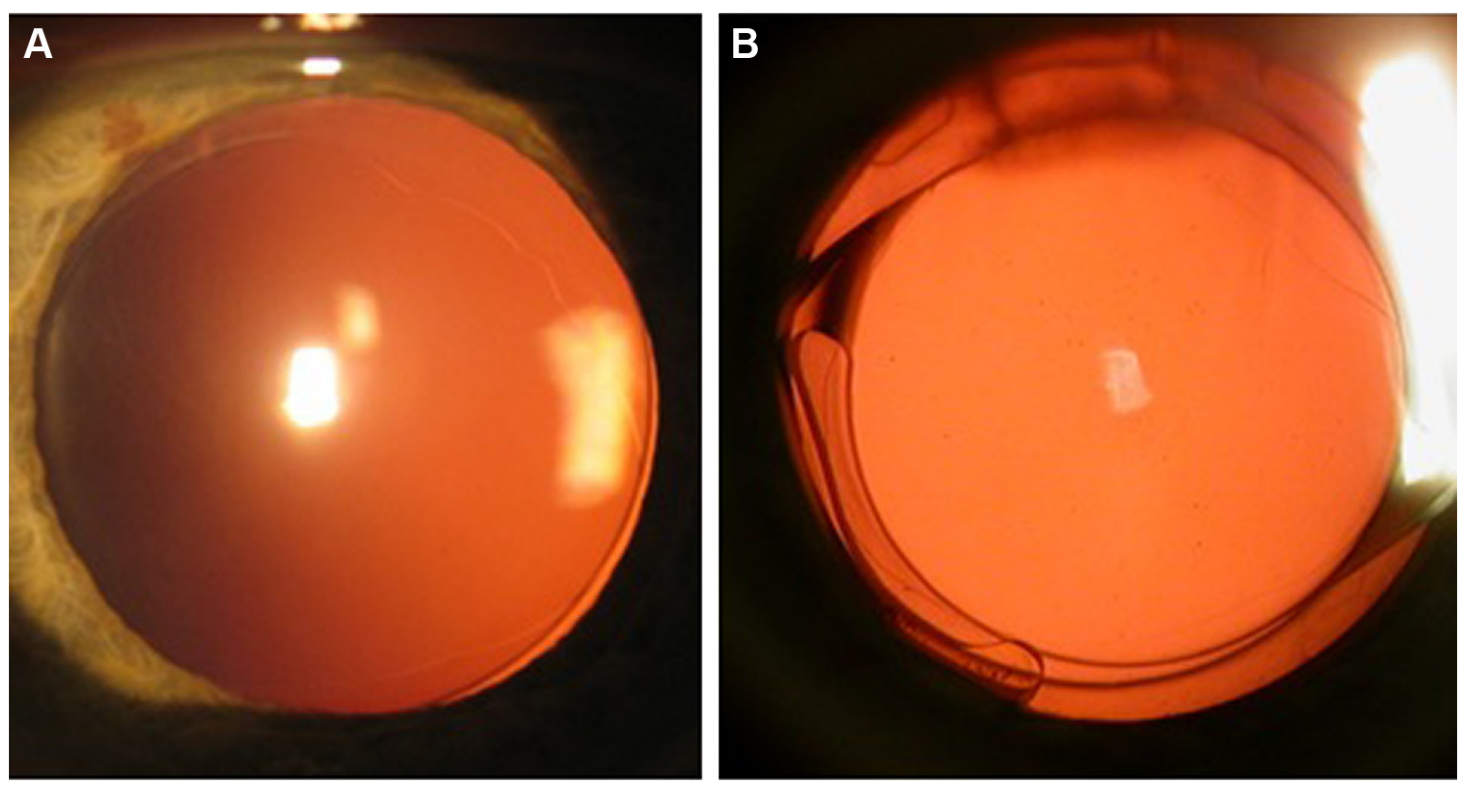

Figure 6 The slit-lamp retroillumination photos before $(\mathbf{A})$ and 4 months after the IOL exchange (B). Abbreviation: IOL, intraocular lens. 

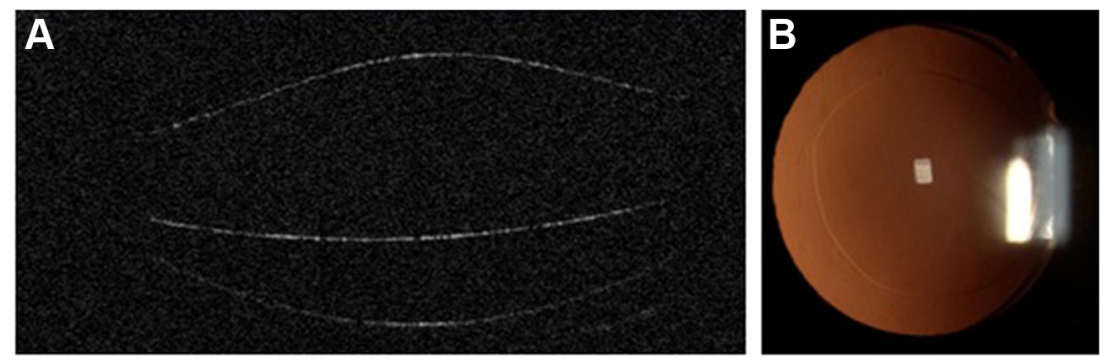

Figure 7 The anterior segment optical coherence tomography photo (A) and slit-lamp photo (B) of clear anterior capsule showing the distance between IOL optic and posterior capsule.

Abbreviation: IOL, intraocular lens.

by Nagamoto et al. ${ }^{15}$ The CAPR is a closed, acrylic ring, with four grooves for IOL loops fixation and four holes for $\mathrm{AH}$ circulation. The study results showed that the use of CAPR can inhibit PCO formation in rabbit eyes when anteroposterior capsular separation and $\mathrm{AH}$ circulation were obtained. The thickness of our ring is sufficient to prevent anteroposterior capsular adhesion and it simultaneously allows $\mathrm{AH}$ circulation in the capsular bag. It could also be possible that the adhesion prevention plays a role in inhibition of both $\mathrm{PCO}$ and ACO (Figure 7).

The sharp-edge posterior optic IOLs are more efficient in preventing PCO than round-edge IOLs. The exact mechanism, by which the square edge influences the LECs migration and therefore PCO development, remains subject of debate. The concept of barrier effect on cellular migration was first explained through the phenomenon of contact inhibition induced by capsular bending. ${ }^{16,17}$ However, another theory was presented supporting the fact that the square edge provides the point of maximum pressure on the posterior capsule, thus the pressure exerted by the square edge prevents cellular migration rather than contact inhibition. ${ }^{18,19}$ The observation made through our experience is that, although our thick endocapsular ring has blunt and round edges, it can still inhibit the migration of LECs and impede the development of PCO. It would seem that contact or pressure of the ring on the capsular bag fornix (equator) may be sufficient to inhibit the migration of LECs.

The placement of the IOL haptics into the ring groove is very important not only for good IOL centration but also for keeping the IOL optics and capsule leaves separated. We observed the disruption of the barrier effect of the ring in the patient with tilted IOL, which resulted in the migration of LECs and formation of PCO (Figure 8). It is interesting to note that migration of LECs continued through the space between the IOL and capsule, but only to the point where the IOL and posterior capsule make contact, as suggested by the theory "no space, no cells."

The drawback of closed endocapsular rings is that they cannot fully adapt to different diameters of capsular bags. On the other hand, an open-ring design of the endocapsular ring apparently suits capsular bags of different size. However, the weakness of this design is a ring gap, which may allow the LECs migration and PCO development, as we noticed in one eye. Although this local PCO did not spread during the last 12 months, it will be interesting to follow its further course.

It is important that no method of PCO prevention should place limitations on a surgeon regarding the IOL type selection. We believe that a great advantage of this endocapsular ring is its compatibility with different types of IOLs. We used

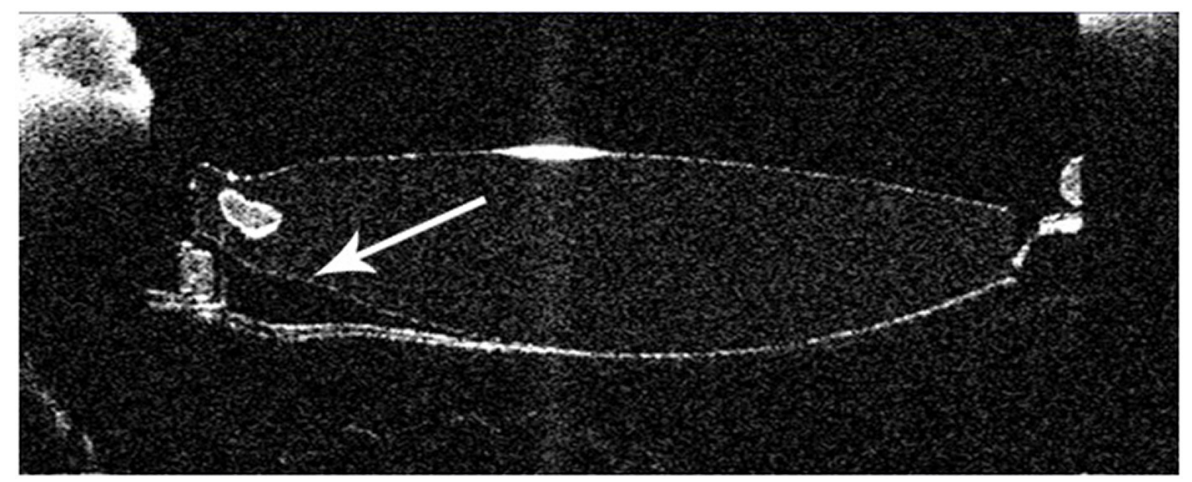

Figure 8 Anterior segment optical coherence tomography photo showing disrupted barrier effect of the ring (arrow) and opacification of the posterior capsule. 

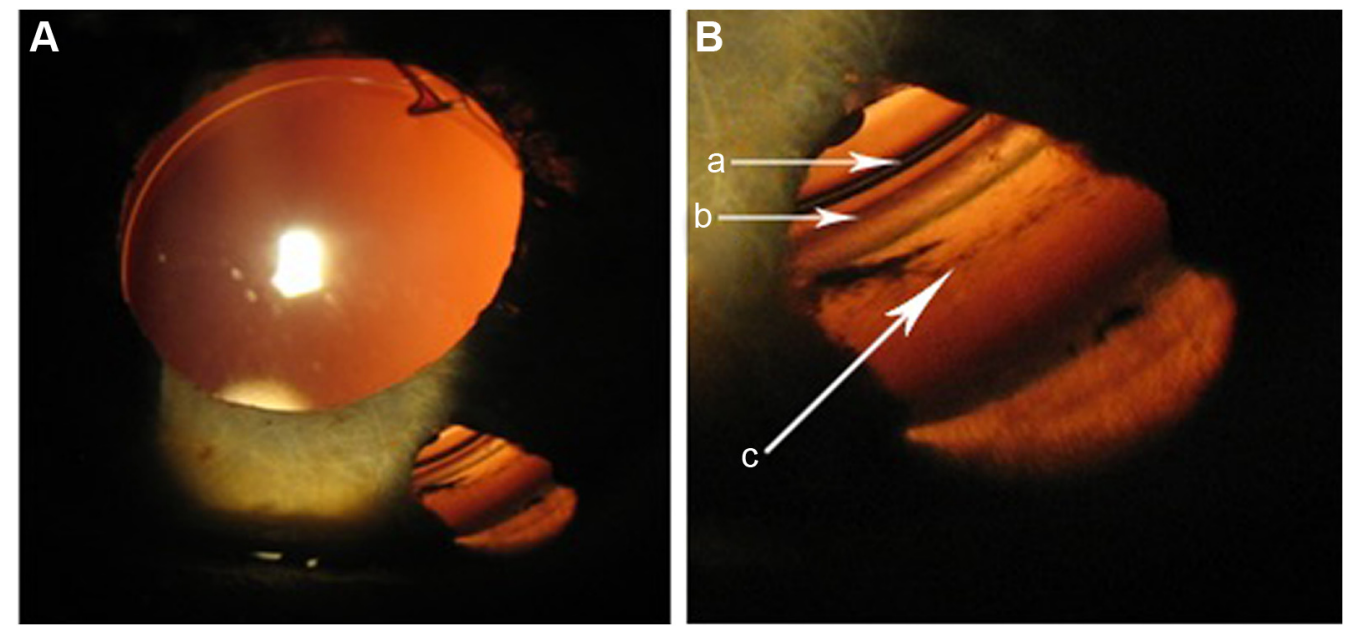

Figure 9 The slit-lamp photo of the peripheral capsule with the ring and Zinn zonules through iridotomy in a patient with previous phakic IOL implantation surgery (a - IOL rim, b - the ring groove, c - anterior capsule zonule attachment). Image $\mathbf{B}$ is a higher magnification of the lower right-hand part of image $\mathbf{A}$.

Abbreviation: IOL, intraocular lens.

six different types of IOLs, which were successfully placed into the capsular bag inside the ring.

This open endocapsular ring is functionally similar to other CTRs. Its ring shape allows Zinn ligament forces to spread evenly, providing a better stability and centration of the IOL. Furthermore, due to its shape, it may function as a peripheral capsular reconstructor with complete anatomical fitting (Figure 9). The standard slim CTRs, though efficient in maintaining IOL centration, do not prevent significantly the migration of LECs. ${ }^{11}$ It is our impression from the slit-lamp examination of our patients that good IOL centration was achieved, but a study of evaluation in an objective manner should be conducted in the future.

\section{Conclusion}

The results of this pilot study suggest that a new design of a thick endocapsular ring may play an important role in the inhibition of PCO and ACO. Although the 3-year results are encouraging, a randomized prospective fellow-eye study with long-term follow-up and a control group is needed for more certain conclusions.

CTRs have well-established use for management of zonular weakness and dehiscence, enhancement of IOL centration, and capsule stabilization. The new design of a thick endocapsular ring may also, in addition to functional abilities of traditional CTRs, play a significant role in the prevention of $\mathrm{ACO}$ and $\mathrm{PCO}$.

\section{Disclosure}

Dr Pallikaris has a pending patent for peripheral capsule reconstructor and declares no other conflicts of interest in this work. The remaining authors have no financial or proprietary interest in any material or method presented herein and declare no conflicts of interest in this work.

\section{References}

1. Apple DJ, Solomon KD, Tetz MR, et al. Posterior capsule opacification. Surv Ophthalmol. 1992;37(2):73-116.

2. Schaumberg DA, Dana MR, Christen WG, Glynn RJ. A systematic overview of the incidence of posterior capsule opacification. Ophthalmology. 1998;105(7):1213-1221.

3. Pandey SK, Apple DJ, Werner L, Maloof AJ, Milverton EJ. Posterior capsule opacification: a review of the aetiopathogenesis, experimental and clinical studies and factors for prevention. Indian J Ophthalmol. 2004;52(2):99-112.

4. Holweger RR, Marefat B. Intraocular pressure change after neodymium: YAG capsulotomy. J Cataract Refract Surg. 1997;23(1):115-121.

5. Koch DD, Liu JF, Gill EP, Parke DW. Axial myopia increases the risk of retinal complications after neodymium-YAG laser posterior capsulotomy. Arch Ophthalmol. 1989;107(7):986-990.

6. Aslam TM, Devlin H, Dhillon B. Use of Nd:YAG laser capsulotomy. Surv Ophthalmol. 2003;48(6):594-612.

7. Trinavarat A, Atchaneeyasakul L, Udompunturak S. Neodymium:YAG laser damage threshold of foldable intraocular lenses. $J$ Cataract Refract Surg. 2001;27(5):775-780.

8. Wormstone IM, Wang L, Liu CS. Posterior capsule opacification. Exp Eye Res. 2009;88(2):257-269.

9. Clark DS. Posterior capsule opacification. Curr Opin Ophthalmol. 2000;11(1):56-64.

10. Hara T, Hara T, Yamada Y. "Equator ring" for maintenance of the completely circular contour of the capsular bag equator after cataract removal. Ophthalmic Surg. 1991;22(6):358-359.

11. Menapace R, Findl O, Georgopoulos M, Rainer G, Vass C, Schmetterer K. The capsular tension ring: designs, applications, and techniques. J Cataract Refract Surg. 2000;26(6):898-912.

12. Menapace R, Sacu S, Georgopoulos M, Findl O, Rainer G, Nishi O. Efficacy and safety of capsular bending ring implantation to prevent posterior capsule opacification: three-year results of a randomized clinical trial. J Cataract Refract Surg. 2008;34(8):1318-1328.

13. Hara T, Hara T, Sakanishi K, Yamada Y. Efficacy of equator rings in an experimental rabbit study. Arch Ophthalmol. 1995;113(8):1060-1065.

14. Hara T, Hara T, Narita M, Hashimoto T, Motoyama Y, Hara T. Long-term study of posterior capsular opacification prevention with endocapsular equator rings in humans. Arch Ophthalmol. 2011;129(7): 855-863. 
15. Nagamoto T, Tanaka N, Fujiwara T. Inhibition of posterior capsule opacification by a capsular adhesion-preventing ring. Arch Ophthalmol. 2009;127(4):471-474.

16. Nishi O, Nishi K, Sakanishi K. Inhibition of migrating lens epithelial cells at the capsular bend created by the rectangular optic edge of a posterior chamber intraocular lens. Ophthalmic Surg Lasers. 1998;29(7): 587-594.

17. Nishi O, Yamamoto N, Nishi K, Nishi Y. Contact inhibition of migrating lens epithelial cells at the capsular bend created by a sharp-edged intraocular lens after cataract surgery. J Cataract Refract Surg. 2007;33(6): 1065-1070.
18. Boyce JF, Bhermi GS, Spalton DJ, El-Osta AR. Mathematical modeling of the forces between an intraocular lens and the capsule. J Cataract Refract Surg. 2000;28(10):1853-1859.

19. Nagamoto T, Fujiwara T. Inhibition of lens epithelial cell migration at the intraocular lens optic edge: role of capsule bending and contact pressure. J Cataract Refract Surg. 2003;29(8):1605-1612.

\section{Publish your work in this journal}

Clinical Ophthalmology is an international, peer-reviewed journal covering all subspecialties within ophthalmology. Key topics include: Optometry; Visual science; Pharmacology and drug therapy in eye diseases; Basic Sciences; Primary and Secondary eye care; Patient Safety and Quality of Care Improvements. This journal is indexed on

\section{Dovepress}

PubMed Central and CAS, and is the official journal of The Society of Clinical Ophthalmology (SCO). The manuscript management system is completely online and includes a very quick and fair peer-review system, which is all easy to use. Visit http://www.dovepress.com/ testimonials.php to read real quotes from published authors. 